\title{
Fracture Mechanics for Electroactive Materials
}

\author{
Robert M. McMeeking \\ Department of Mechanical Engineering \\ University of California, Santa Barbara, California 93106 USA
}

\section{Extended abstract}

Electroactive materials include piezoelectrics, ferroelectrics and electroactive polymers. In the simplest form, this class of materials also includes isotropic dielectrics where there is minimal coupling between the mechanics and electrostatics. Indeed, electroactive polymers can be isotropic. Then they derive their useful properties of electrically stimulated actuation from their low mechanical stiffness and then large actuation strains occur when electric fields are applied. In the presentation, a consistent energetic formulation of boundary value problems for electroactive materials is formulated, including the electrostatic stress. This step is important because a correct energy balance can only be achieved after the proper formulation is obtained. The energy release rate for cracks in electroactive materials is then calculated and the J-integral for these systems obtained. The results are specialized to simple isotropic dielectrics, electroactive polymers, piezoelectrics and ferroelectrics. Fracture models are then considered for each type of material, including brittle failure concepts for simple dielectrics and piezoelectrics, switching zone effects in ferroelectrics and energy balance models for electroactive polymers. The outcome is a comprehensive and consistent fracture mechanics for electroactive materials. The concepts can also be extended to magnetoactive materials.

With the electric field given by $E_{i}$, the material polarization by $P_{i}$, the Cauchy stress by $\sigma_{i j}$, the material velocity by $v_{i}$ and position by $x_{i}$, the rate of internal work in a volume $V$ containing material is given by [1]

$$
\begin{aligned}
\frac{\mathrm{d} W}{\mathrm{~d} t} & =\frac{\mathrm{d}}{\mathrm{d} t} \int_{V} \frac{1}{2} \kappa_{0} E_{i} E_{i} \mathrm{~d} V+\frac{\mathrm{d}}{\mathrm{d} t} \int_{V} \frac{1}{2} \rho v_{i} v_{i} \mathrm{~d} V \\
& +\int_{V}\left[E_{i} \frac{\mathrm{d} P_{i}}{\mathrm{~d} t}+\left(\sigma_{j i}+\sigma_{j i}^{M}-\hat{\sigma}_{j i}^{M}-E_{i} P_{j}+E_{k} P_{k} \delta_{i j}\right) \frac{\partial v_{i}}{\partial x_{j}}\right] \mathrm{d} V
\end{aligned}
$$

where

$$
\hat{\sigma}_{i j}^{M}=\kappa_{o}\left(E_{i} E_{j}-\frac{1}{2} E_{k} E_{k} \delta_{i j}\right)
$$

is the Maxwell stress at the specified electric field for free space absent any material, the superscript $M$ on stress indicates the material Maxwell stress, $\kappa_{o}$ is the permittivity of free space and $\rho$ is the material density. This external work rate is 


$$
\frac{\mathrm{d} W}{\mathrm{~d} t}=\int_{V} b_{i} v_{i} \mathrm{~d} V+\int_{S} T_{i} v_{i} \mathrm{~d} S+\int_{V} \phi \frac{\mathrm{d}}{\mathrm{d} t}(q \mathrm{~d} V)+\int_{S} \phi \frac{\mathrm{d}}{\mathrm{d} t}(\omega \mathrm{d} S)
$$

where $b_{i}$ is the mechanical body force per unit volume (e.g. gravity), $T_{i}$ is the mechanical traction on surfaces $S, \phi$ is the electrical potential, $q$ is the extrinsic charge density per unit volume and $\omega$ is the extrinsic charge per unit area of $S$.

In the quasi-static limit, Maxwell's laws state that the electric field must be curl-free and Gauss' law states that the divergence of the electric displacement must be equal to the volume density of free charge. Therefore,

$$
\begin{aligned}
& \in_{i j k} \frac{\partial E_{j}}{\partial x_{k}}=0 \Rightarrow E_{i}=-\frac{\partial \phi}{\partial x_{i}} \\
& \frac{\partial D_{i}}{\partial x_{i}}=q \text { in } V \\
& \left\|D_{i} n_{i}\right\|=\omega \text { on } S
\end{aligned}
$$

Here $D_{i}$ is the electric displacement, $n_{i}$ are the Cartesian components of the unit normal to the surface $S$ pointing from the "-" side of the surface out towards the "+" side, and $\in_{i j k}$ are the components of the permutation symbol. Then the notation \|\| represents the difference or jump in the included quantity across the surface $S$ such that

$$
\left\|D_{i}\right\|=D_{i}^{+}-D_{i}^{-}
$$

Furthermore, the electric displacement can be decomposed into two parts such that

$$
D_{i}=\kappa_{0} E_{i}+P_{i}
$$

where $\kappa_{0}$ is the dielectric permittivity of free space.

The principles of conservation of linear and angular momentum yield

$$
\frac{\partial \sigma_{j i}}{\partial x_{j}}+\frac{\partial \sigma_{j i}^{M}}{\partial x_{j}}+b_{i}=\rho \frac{\mathrm{d} v_{i}}{\mathrm{~d} t} \text { in } V
$$

and

$$
\sigma_{j i}+\sigma_{j i}^{M}=\sigma_{i j}+\sigma_{i j}^{M} \text { in } V
$$

Thus, for the balance of angular momentum to be satisfied, the sum of the Cauchy and Maxwell stresses must be symmetric. This requirement reflects the fact that moments 
due to mechanical body forces and inertia can be assumed, as usual, to be $2^{\text {nd }}$ order but we must allow for the possibility that electric effects induce $1^{\text {st }}$ order moments, e.g. due to electric fields acting on dipoles in the material. Therefore it is possible that both the Maxwell and Cauchy stress tensors can be non-symmetric. In order to satisfy the principle of conservation of linear momentum for a small surface element, the Cauchy stress in the material, $\sigma_{i j}$, must balance the total surface traction such that

$$
T_{i}=-n_{j}\left\|\sigma_{j i}+\sigma_{j i}^{M}\right\|
$$

That is, the Cauchy stress difference across a surface must balance both the electrical and mechanical surface tractions where the difference in Maxwell stress across the surface represents the electrical traction. Note that the Maxwell stress is not zero within a crack. Thus, there are generally electrical tractions at the surface of a crack and it is critical to take these into account to ensure that energy balance is properly handled.

For conservative materials, energy balance as in Eq. (1-3) taking into account Eq. (411) leads to the constitutive relationship for stress

$$
\sigma_{j i}+\sigma_{j i}^{M}-\hat{\sigma}_{j i}^{M}-E_{i} P_{j}+E_{k} P_{k} \delta_{i j}=\rho \frac{\partial \psi}{\partial F_{i k}} F_{j k}
$$

where $\psi$ is the stored energy in the material per unit mass and $F_{i j}$ is the deformation gradient. In addition, the energy balance provides the constitutive law for electric field

$$
E_{i}=\rho \frac{\partial \psi}{\partial P_{i}}
$$

The formulation above is a consistent treatment of the mechanics and electrostatics for electroactive materials. With appropriate forms of $\psi$, constitutive laws suitable for isotropic dielectrics, piezoelectrics, ferroelectrics and electroactive polymers can be developed. Crack problems can then be solved and results obtained for the energy release rate. In parallel, the J-integral can be formulated to encompass the same outcome and a full set of tools for the fracture mechanics of electroactive materials is then available. For example, in an infinite, linear, isotropic, brittle dielectric having a through crack of length $2 a$, an applied stress of $\sigma_{A}$ and an applied electric field of $E_{A}$, the electric field in the crack is $\kappa E_{A} / \kappa_{o}$ where $\kappa$ is the dielectric permittivity of the material. Due to the applied load and the Maxwell traction in the crack, the Mode I stress intensity factor is given by

$$
K_{I}=\left[\sigma_{A}-\frac{\kappa^{2} E_{A}^{2}}{2 \kappa_{o}}\right] \sqrt{\pi a}
$$

The energy release rate and J-integral is 


$$
G=\frac{1}{Y^{\prime}}\left[\sigma_{A}-\frac{\kappa^{2} E_{A}^{2}}{2 \kappa_{o}}\right]^{2} \pi a
$$

where $Y^{\prime}$ is the elastic modulus for plane strain deformation. A simple brittle fracture criterion would involve a critical value for the energy release rate, thereby defining the role of the electric field in inhibiting crack growth. These results are readily extended to piezoelectrics, ferroelectrics with switching zone toughening and electroactive polymers.

[1] R.M. McMeeking and C.M. Landis, "Electrostatic forces and stored energy for deformable dielectric materials,” submitted to Journal of Applied Mechanics, 2004. 